19 Revue d'histoire du XIXe siècle

Société d'histoire de la révolution de 1848 et des

révolutions du XIXe siècle

55 | 2017

Les écoles du peuple à l'ère des révolutions

(1815-1880)

Éric ANCEAU, L'Empire libéral. Tome I : Genèse, avènement, réalisations. Tome II : Menaces, chute, postérité

Paris, Éditions SPM, 2017, 1425 p., $76 €$

Jean-Claude Caron

\title{
OpenEdition
}

Journals

Édition électronique

URL : http://journals.openedition.org/rh19/5366

DOI : $10.4000 /$ rh 19.5366

ISSN : $1777-5329$

Éditeur

La Société de 1848

Édition imprimée

Date de publication : 1 décembre 2017

Pagination : 227-230

ISSN : 1265-1354

Référence électronique

Jean-Claude Caron, «Éric ANCEAU, L'Empire libéral. Tome I: Genèse, avènement, réalisations. Tome II Menaces, chute, postérité », Revue d'histoire du XIXe siècle [En ligne], 55 | 2017, mis en ligne le 01 décembre 2017, consulté le 05 janvier 2021. URL : http://journals.openedition.org/rh19/5366 ; DOI : https://doi.org/10.4000/rh19.5366

Ce document a été généré automatiquement le 5 janvier 2021.

Tous droits réservés 


\section{Éric ANCEAU, L'Empire libéral. Tome I : Genèse, avènement, réalisations. Tome II : Menaces, chute, postérité}

Paris, Éditions SPM, 2017, 1425 p., $76 €$

Jean-Claude Caron

\section{RÉFÉRENCE}

Éric ANCEAU, L'Empire libéral. Tome I : Genèse, avènement, réalisations. Tome II :

Menaces, chute, postérité, Paris, Éditions SPM, 2017, 1425 p., $76 €$

1 Le caractère déraisonnable de ces deux tomes est d'autant plus jubilatoire que l'Empire libéral dont il est ici question ne couvre - théoriquement - qu'une période de neuf mois. En effet, Éric Anceau réduit la notion au gouvernement du 2 janvier 1870, distinguant le lent phénomène de libéralisation du Second Empire entamé en 1860 de sa traduction concrète en un bref régime libéral. C'est à cette genèse que s'attaque l'auteur, adepte d'une "histoire politique totale", dans sa première partie de 400 pages. Puis il étudie un « état de grâce » dont il montre le caractère très relatif dans les 300 pages suivantes. Enfin, il analyse les raisons de sa « Berezina » ambiguë dans les dernières 370 pages. Précisons que l'ouvrage comporte également de riches annexes, plus de 80 pages de sources et près de 100 de bibliographie, ainsi qu'un indispensable index. Quant à l'objectif d'Éric Anceau, il est de fournir une analyse très documentée de son sujet, afin de fournir une vision objective, juste, équilibrée de la période envisagée. Reste à définir ce que ces adjectifs signifient, à considérer que l'écriture d'une histoire objective relève du possible, y compris chez les historiens qui ont entrepris non une réévaluation, mais bien une réhabilitation du Second Empire. Pour le dire autrement, par ses origines mais autant par sa fin, la période reste un moment pas tout-à-fait 
refroidi du récit national. Mais aussi un moment dont l'auteur souligne à juste titre l'intérêt du point de vue des cinq concepts qu'il mobilise pour l'aborder: "modernisation de la société ", "nationalisation des masses", "politisation", « écosystèmes politiques », « transition politique ».

2 La très ambitieuse première partie, savante synthèse, considère de fait la totalité du Second Empire, dans toutes ses dimensions, privilégiant toutefois les débats politiques et constitutionnels, les rendez-vous électoraux, au détriment notamment de l'économie, rapidement évoquée, mais aussi de la guerre ou de la culture. Les débats sur les réformes constitutionnelles y sont finement analysés, en particulier les divergences d'opinion qu'elles entrainent parmi les opposants au régime. On apprécie également la dimension nationale, et pas seulement parisienne, de l'étude, qui laisse une grande place aux départements et aux populations rurales, fruit d'un intense travail de collecte dans 82 centres d'archives. On lira avec profit les pages consacrées aux conseils généraux et municipaux, au débat sur la décentralisation, au fonctionnement de l'administration, mais aussi le très suggestif chapitre traitant par une approche prosopographique, sociale et générationnelle, la question des élites au printemps 1869. L'importance de cette année dans l'évolution du régime est corroborée par les élections de 1869, analysées en détail. Cette première partie s'achève par un portrait d'Émile Ollivier : portrait assez peu critique, du reste, de cette réincarnation d'un juste-milieu libéral occupant l'espace entre la démocratie républicaine émergeant avec de nouvelles figures (Gambetta étant la plus connue) et un bonapartisme rouhérien en perte de vitesse.

Dans la deuxième partie, Éric Anceau alterne les chapitres dynamiques - ainsi de l'avènement du gouvernement du 2-Janvier et des réactions qu'il suscite, mais aussi le choc quasi immédiat avec l'affaire Victor Noir - et les chapitres analytiques. Parmi ces derniers, on signalera tout l'intérêt de ceux consacrés au personnel de l'Empire libéral et à l'organisation des grandes commissions. Clairement, Ollivier fut confronté en permanence à la question de sa légitimité et soumis à des pressions contradictoires venant tant de la droite que de la gauche, avec Napoléon III comme arbitre. Il lui fallut gérer aussi les effets, pas nécessairement négatifs pour lui, de l'abandon de la candidature officielle. Le corps préfectoral fut profondément remanié, même si, comme le souligne l'auteur, qui étend son enquête aux sous-préfets, secrétaires généraux et conseillers de préfecture, peu d'hommes neufs furent appelés. Ollivier fut plus prudent avec la magistrature et l'épiscopat. Quant aux grandes commissions parlementaires, dont l'étude, véritablement novatrice, éclaire le fonctionnement des institutions parlementaires, Éric Anceau en donne un panorama très complet qui constitue l'un des temps forts de l'ouvrage. Enjeux politiques, sociaux, économiques s'affrontent autour des transports, de la liberté de l'enseignement supérieur, de l'administration de Paris, du régime économique de la France, de la décentralisation, etc. Ces débats à la fois techniques et politiques, menés également dans des commissions extra-parlementaires, permettent de réintégrer dans le jeu politique des opposants qui, par ce biais, opèrent un ralliement de facto au régime, tels Odilon Barrot ou Guizot, même si d'importantes personnalités légitimistes, orléanistes et républicaines refusèrent d'en être afin de ne pas se compromettre (Falloux, Dufaure, Simon, Grévy, etc.). De cette accumulation de débats, accompagnés d'une montagne de rapports, il ne sortit en définitive que peu de choses, moins encore pour les commissions extra-parlementaires, purement consultatives, comme celle sur la décentralisation. Plus globalement et au seul plan de 
l'action gouvernementale, il apparait qu'Ollivier, à la fois par la volonté de conserver une majorité et par un caractère pour le moins prudent, se pose davantage en arbitre qu'en chef. Le pouvait-il réellement, du reste, au vu de la fragilité persistante de sa position dans un système qui, bien que profondément réformé, ne peut encore être considéré comme parlementaire ? Pourtant face à la dégradation du climat social et à une contestation plus radicale du régime avec l'avènement d'un socialisme révolutionnaire, Ollivier propose de nouvelles réformes constitutionnelles. Au point d'arrivée, la constitution de 1870 est analysée comme «le fruit hybride d'un compromis ", aboutissant non à un régime parlementaire, mais à un gouvernement représentatif ou mixte, mâtiné d'une dose plébiscitaire persistante: ce qu'illustre le recours au plébiscite du 8 mai que l'auteur étudie en détail, depuis le débat sur son principe même, sa formulation, son organisation, la mise en œuvre d'une puissante machine plébiscitaire, jusqu'à l'action des opposants (167 comités anti-plébiscitaires furent mis en place, essentiellement par les républicains), les résultats et leurs conséquences. Comme en d'autres cas, la question posée par le plébiscite (approuver les réformes constitutionnelles) passe au second rang, celle de la légitimation du souverain et du régime prenant le dessus. On observe par ailleurs que, en dehors du camp républicain, la quasi-totalité des courants politiques sont divisés sur la position à adopter (oui, non ou abstention).

4 La troisième partie donne l'occasion à Éric Anceau de dresser un tableau très précis du climat de tension extrême qui entoure le gouvernement du 2-Janvier à partir du moment où la France entre dans une logique guerrière face à la Prusse. Il établit les responsabilités de Napoléon III et d'ollivier: si le premier, physiquement diminué, entend à nouveau faire de la guerre un outil de politique nationale comme internationale, le second se laisse embarquer dans une entreprise contraire à ses idées. L'auteur propose par ailleurs un état très complet des réactions de l'opinion publique, globalement favorable à l'entrée en guerre. Mais la rapidité et l'ampleur des défaites militaires entraînèrent une vive inquiétude donnant corps à toutes les rumeurs, et provoquèrent de violentes manifestations - et accessoirement la démission d'ollivier, devenu l'homme "au cœur léger». C'est dans ce contexte très particulier que sont organisées les élections municipales marquées par une poussée républicaine. Éric Anceau montre combien la mise en place d'un pouvoir bicéphale - l'empereur conduisant la guerre sur le terrain, l'impératrice exerçant la régence à Paris - a très vite rendu ingérable la situation, conduisant au 4 septembre. L'auteur suit ensuite le parcours d'ollivier après sa démission et la chute de l'Empire : l'exil d'abord, puis le retour avec l'ajournement de sa réception à l'Académie française. Car l'homme reste marqué par son passé indélébile - Cassagnac le compare aimablement à un parricide et échoue à retrouver une position politique. Un parcours finalement assez semblable à celui d'un Guizot - qu'il déteste - et d'un Lamartine - qu'il admire. Rallié à la République, sans renier son idéal d'un exécutif fort, il se livre alors à des travaux d'écriture, dont les dix-sept volumes du monumental Empire libéral. Plus méconnu est le parcours d'autres figures de l'Empire libéral, peu d'entre elles réussissant leur retour en politique, sauf pour des fonctions subalternes. L'épuration des grands corps fut très inégale: si le pragmatisme l'emporta souvent, les ambassadeurs, les préfets et les procureurs-généraux - mais aussi les juges de paix - furent fortement touchés dès 1870 ; et la tendance à la républicanisation de la fonction publique se poursuivit après l'élection du 8 février 1871, non sans à-coups au vu des aléas politiques des premières années de la Troisième République. Dans le dernier chapitre, "Contre-modèle et 
héritage inavouable ", l'auteur passe en revue les très nombreuses analyses de tous bords politiques qui, tentant de comprendre les origines du mal - le surgissement de «l'année terrible »-, proposent des remèdes, non sans contradictions parfois. Du reste, les pratiques républicaines de gouvernement ne sont pas sans parenté avec certaines pratiques impériales. Au cœur de ce qui apparaît comme une sorte d'histoire totale de la République naissante, Éric Anceau met certes en exergue l'affirmation d'un monisme parlementaire tout-puissant, aux antipodes donc du Second Empire. Mais il rappelle que, loin de se situer toujours dans la rupture (l'Empire libéral comme contre-modèle), le régime républicain prend parfois en compte un héritage inavouable. C'est en particulier le cas, souligne l'auteur, dans «le rapport de l'État aux corps intermédiaires ", trahissant une "forme de jacobino-bonapartisme atténué ».

5 La conclusion en forme de synthèse de l'ouvrage laisse transparaitre un bilan plutôt positif de l'Empire libéral, avec en particulier un approfondissement de l'apprentissage du suffrage universel. Éric Anceau pèse soigneusement chacun de ses mots, soucieux de répondre au principe d'objectivité mis en exergue dans l'introduction. Affirmant que la République "dériva » vers le régime d'assemblée, il en déduit que «la souveraineté populaire fut largement vidée de son sens au profit d'une souveraineté nationale inscrite dans une démocratie représentative et une République légicentrée où l'essentiel du pouvoir relevait du Parlement»: soit, mais on peut rappeler que cela avait déjà été le cas avec la Seconde République, et l'on n'ose imaginer que ce constat justement critique soit un éloge caché d'une démocratie autoritaire incarnée par le césarisme bonapartiste, persistant sous l'Empire libéral. Le rapprochement final avec la République gaullienne est intéressant, mais assez rapide. Plus largement l'auteur inscrit son propos dans une réflexion globale sur la question - pour le moins actuelle, à en croire les propos de l'actuel président de la République - de la réforme en France, ce pays marqué par une "schizophrénie entre une confiance absolue et un abattement démesuré ». Il en appelle finalement à une troisième voie entre « un modèle républicain mythifié » et « un modèle démocratique à l'anglo-saxonne » : soit un régime fondé sur « la pluralité, la complexité et le libre examen humaniste et ouvert de ce qu'il est souhaitable et possible de faire, en usant sa réserve de puissance pour agir à bon escient après avoir identifié la (ou les) réforme(s) qui serai(en)t décisive(s) ». À sa manière, Émile Ollivier entendait incarner cette troisième voie : mais la brièveté de son expérience gouvernementale, même en tenant compte des circonstances particulières qui l'expliquent, interroge sur la possibilité même de son existence. 